\title{
Software engineering principles to improve quality and performance of $\mathbf{R}$ software
}

\author{
Seth Russell Corresp., 1 , Tellen D Bennett ${ }^{1,2}$, Debashis Ghosh ${ }^{1,3}$ \\ 1 University of Colorado Data Science to Patient Value, University of Colorado Anschutz Medical Campus, Aurora, CO, United States of America \\ 2 Pediatric Critical Care, University of Colorado School of Medicine, Aurora, CO, United States of America \\ 3 Department of Biostatistics and Informatics, Colorado School of Public Health, Aurora, CO, United States of America \\ Corresponding Author: Seth Russell \\ Email address: seth.russell@ucdenver.edu
}

Today's computational researchers are expected to be highly proficient in using software to solve a wide range of problems ranging from processing large datasets to developing personalized treatment strategies from a growing range of options. Researchers are well versed in their own field, but may lack formal training and appropriate mentorship in software engineering principles. Two major themes not covered in most university coursework nor current literature are software testing and software optimization. Through a survey of all currently available Comprehensive R Archive Network (CRAN) packages, we show that reproducible and replicable software tests are frequently not available and that many packages do not appear to employ software performance and optimization tools and techniques. Through use of examples from an existing $\mathrm{R}$ package, we demonstrate powerful testing and optimization techniques that can improve the quality of any researcher's software. 


\title{
1 Software engineering principles to improve quality
}

\section{2 and performance of $\mathbf{R}$ software}

3

4

5

6

7

8 9

12

\author{
Seth Russell ${ }^{1}$, Tellen D. Bennett ${ }^{1,2}$, Debashis Ghosh ${ }^{1,3}$
}

${ }^{1}$ University of Colorado Data Science to Patient Value (D2V), Anschutz Medical Campus, Aurora, CO, United States of America

2 Pediatric Critical Care, University of Colorado School of Medicine, Aurora, CO, United States of America

${ }^{3}$ Biostatistics and Informatics, Colorado School of Public Health, Aurora, CO, United States of America

Corresponding Author:

Seth Russell

CU Data Science to Patient Value

13199 E. Montview Blvd, Suite 210-15

Aurora, CO 80045 USA

Email address: seth.russell@ucdenver.edu

\section{ABSTRACT}

\section{INTRODUCTION}

Writing scientific software has progressed from the work of early pioneers to a range of computer professionals, computational researchers, and self-taught individuals. The educational discipline of computer science, standardized many years ago through recommendations from the Association for Computing Machinery (ACM) (Atchison et al., 1968), has grown in breadth and depth over many years. Software engineering, a discipline within computer science, "seeks to develop and use systematic models and reliable techniques to produce high-quality software. These software engineering concerns extend from theory and principles to development practices that are most visible to those outside the discipline." (The Joint Task Force on Computing Curricula, 2015)

As they gain sophistication, computational researchers, statisticians, and similar professionals need to advance their skills by adopting principles of software engineering. Wilson et al. identified 8 key areas where scientists can benefit from software engineering best practices (Wilson et al., 2014). The term "best" as referenced in the previous cited work and others cited later refers to expert consensus based on knowledge and observational reporting of results from application of the practices. They provide a high-level description of 8 important 
40 principles of software engineering that should "reduce the number of errors in scientific

41 software, make it easier to reuse, and save the authors of the software time and effort that can

42 used for focusing on the underlying scientific questions." While their principles are still relevant

43 and important today, there has not been enough progress in this endeavor, especially with respect

44 to software testing and software optimization principles (Wilson, 2016; Nolan \& Padilla-Parra,

45 2017).

46

47

The ACM/Institute of Electrical and Electronics Engineers (IEEE) recommendations for an undergraduate degree in software engineering describe a range of coursework and learning objectives. Their guidelines call out 10 specific knowledge areas that should be part of or guide all software engineering coursework. The major areas are: computer science fundamentals, math and engineering fundamentals, professional interactions/communication, software modeling, requirement gathering, software design, verification, project processes, quality, and security (The Joint Task Force on Computing Curricula, 2015). These major themes are not covered extensively outside software engineering and include such generally applicable items such as software verification, validation, testing, and computer science fundamentals (e.g. software optimization, modeling, and requirement gathering).

In addition to the need for further training, understanding the software lifecycle is necessary: the process of software development from ideation to delivery of code. The largest component of software's lifecycle is maintenance. Software maintenance costs are large and increasing (Glass, 2001; Dehaghani \& Hajrahimi, 2013; Koskinen, 2015); some put maintenance at $90 \%$ of total software cost. The chief factor in software maintenance cost is the time of the people creating and using the software. From the recent trend on making research results reproducible and replicable, some recommend making code openly available to any who might wish to repeat or further analyze results (Leek \& Peng, 2015). With the development of any software artifact, an important consideration for implementation should be maintenance. As research scientists tend to think of their software products as unique tools that will not be used regularly or for a long period, they often do not consider long term maintenance issues during the development phase (Sandve et al., 2013; Prins et al., 2015). While a rigorous and formal software engineering approach is not well suited to the standard lifecycle of research software (Wilson, 2016), there are many techniques that can help to reduce cost of maintenance and speed development. While best practices such as the use of version control software, open access to data, software, and results are becoming more wide spread, other practices such as testing and optimization need further attention.

In this paper, a brief survey of currently available $\mathrm{R}$ packages from The Comprehensive $\mathrm{R}$ Archive Network (CRAN) will be used to show the continued need for software testing and optimization. Source code for this analysis is freely available at https://github.com/magiclantern/SoftwareEngineeringPrinciples. After the presentation of the current state of R packages, 
80 general advice on software testing and optimization will be presented. The R package "pccc:

81 Pediatric Complex Chronic Conditions" (Feinstein et al., 2018; DeWitt et al., 2017) (pccc),

82 available via CRAN and at https://github.com/CUD2V/pccc, is used for code examples in this

83 article. pccc is a combined $\mathrm{R}$ and $\mathrm{C}++$ implementation of the Pediatric Complex Chronic

84 Conditions software released as part of a series of research papers (Feudtner, Christakis \&

85 Connell, 2000; Feudtner et al., 2014). pccc takes as input a data set containing International

86 Statistical Classification of Diseases and Related Health Problems (ICD) Ninth revision or Tenth

87 revision diagnosis and procedure codes and outputs which if any complex chronic conditions a

88 patient has.

89

90

91

92

93

94

95

96

97

98

99

100

101

102

103

104

105

106

107

108

109

110

111

112

113

114

115

116

117

118

119

\section{ANALYSIS OF R PACKAGES ON CRAN}

\section{TESTING OF R PACKAGES}

In order to estimate the level of testing common among $\mathrm{R}$ software, we analyzed all $\mathrm{R}$ packages available through CRAN. Although Nolan (Nolan \& Padilla-Parra, 2017) performed a similar analysis in the past, due to the rapid change in the CRAN as a whole, a reevaluation is necessary. At the time of Nolan's work, CRAN contained 10084 packages; it now contains 13509. Furthermore, the analysis by Nolan had a few shortcomings that we have addressed in this analysis: there are additional testing frameworks for which we wanted to analyze their usage; not all testing frameworks and R packages store their test code in a directory named "tests"; only packages modified in the past 2 years were reported - there are many commonly used R packages that have not been updated in the last 2 years.

Although we address some shortcomings in analyzing R code for use of testing best practices, our choice of domain for analysis does have some limitations. Not all research software is written in R; for those that do use R, not all software development results in a package published on CRAN. While other software languages have tools for testing, additional research would be needed to evaluate level of testing in those languages to see how it compares to this analysis. Although care has been taken to identify standard testing use cases and practices for $\mathrm{R}$, testing can be performed in-line through use of core functions such as stop() or stopifnot(). Also, developers may have their own test cases they run while developing their software, but did not include them in the package made available on CRAN. Unit tests can be considered executable documentation, a key method of conveying how to use software correctly (Reese, 2018).

Published research that involves software is not as easy to access and evaluate for use of testing code as CRAN packages are. While some journals have standardized means for storing and sharing code, many leave the storing and sharing of code up to the individual author, creating an environment where code analysis would require significant manual effort.

To analyze use of software testing techniques, we evaluated all CRAN packages on two different metrics: 
120

121

122

123

124

125

126

127

128

129

130

131

132

133

134

135

136

137

138

139

140

141

142

143

144

145

146

147

148

149

150

151

152

153

154

155

156

157 158

Metric 1: In the source code of each package, search for non-empty testing directories using the regular expression pattern "[Tt]est $[\wedge /]^{*} / .+$ ". All commonly used R testing packages (those identified for metric 2 ) recommend placing tests in a directory by themselves, which we look for.

Metric 2: Check for stated dependencies on one of the following testing packages: RUnit (Burger, Juenemann \& Koenig, 2015), svUnit (Grosjean, 2014), testit (Xie, 2018), testthat (Wickham, 2011), unitizer (Gaslam, 2017), or unittest (Lentin \& Hennessey, 2017). From the authors of these packages, it is recommended to list dependency (or dependencies) to a testing framework even though standard usage of a package may not require it.

For the testing analysis, we used 2008 as the cutoff year for visualizations due to the low number of packages last updated prior to 2008 .

As shown in Figure 1, the evaluation for the presence of a non-empty testing directory shows that there is an increasing trend in testing R packages, with $44 \%$ of packages updated in 2018 having some tests. Table S1 contains the data used to generate Figure 1.

As shown in Figure 2, reliance upon testing frameworks is increasing over time both in count and as a percentage of all packages. There 16 packages that list dependencies on more than one testing framework ( 9 with dependencies on both RUnit and testthat, 7 with dependencies on both testit and testthat), so the total number of packages shown in the histogram includes 16 that are double counted. Table S2 contains the data used to generate Figure 2.

As the numbers from Metric 1 do not match the numbers of Metric 2, some additional exploration is necessary. There are 884 more packages identified from Metric 1 vs Metric 2. There are 1115 packages that do not list a dependency to a testing framework, but have a testing directory; e.g. the package xlsx (Dragulescu \& Arendt, 2018). Some packages use a testing framework, but do not list it as a dependency; e.g. the package redcapAPI (Nutter \& Lane, 2018). There are also 231 packages that list a testing framework as a dependency, but do not contain a directory with tests. See Supplemental tables S1 and S2 for more details.

\section{OPTIMIZATION OF R PACKAGES}

In order to estimate the level of software optimization common among $\mathrm{R}$ software, we performed an analysis of all $\mathrm{R}$ packages available through CRAN. To analyze the use of software optimization tools and techniques, we evaluated all CRAN packages on two different metrics: 
159

160

161

162

163

164

165

166

167

168

169

170

171

172

173

174

175

176

177

178

179

180

181

182

183

184

185

186

187

188

189

190

191

192

193

194

195

196

197

198

Metric 1: In the source code of each package, search for non-empty src directories using the regular expression pattern $" \operatorname{src}[\wedge /]^{*} / .+"$. By convention, packages using compiled code $(\mathrm{C}, \mathrm{C}++$, Fortran) place those files in a '/src' directory.

Metric 2: Check for stated dependencies on packages that can optimize, scale performance, or evaluate performance of a package. Packages included in analysis are: DSL (Feinerer, Theussl \& Buchta, 2015), Rcpp (Eddelbuettel \& Balamuta, 2017), RcppParallel (Allaire et al., 2018a), Rmpi (Yu, 2002), SparkR (Apache Software Foundation, 2018), batchtools (Bischl et al., 2015), bench (Hester, 2018), benchr (Klevtsov, Antonov \& Upravitelev, 2018), doMC (Calaway, Analytics \& Weston, 2017), doMPI (Weston, 2017), doParallel (Calaway et al., 2018), doSNOW (Calaway, Corporation \& Weston, 2017), foreach (Calaway, Microsoft \& Weston, 2017), future (Bengtsson, 2018), future . apply (Bengtsson \& R Core Team, 2018), microbenchmark (Mersmann, 2018), parallel (R Core Team, 2018), parallelDist (Eckert, 2018), parallelMap (Bischl \& Lang, 2015), partools (Matloff, 2016), profr (Wickham, 2014a), profvis (Chang \& Luraschi, 2018), rbenchmark (Kusnierczyk, Eddelbuettel \& Hasselman, 2012), snow (Tierney et al., 2018), sparklyr (Luraschi et al., 2018), tictoc (Izrailev, 2014).

For the optimization analysis, we used 2008 as the cutoff year for visualizations showing presence of a src directory due to the low number of currently available packages last updated prior to 2008. For optimization related dependencies, in order to aid visual understanding, we used 2009 as the cutoff year and only showed those packages with 15 or greater dependent packages in a given year.

Automatically analyzing software for evidence of optimization has similar difficulties to those mentioned previously related to automatically detecting the use of software testing techniques and tools. The best evidence of software optimization would be in the history of commits, unit tests that time functionality, and package bug reports. While all $\mathrm{R}$ packages have source code available, not all have development history available nor unit tests available. Additionally, a stated dependency on one of the optimization packages listed could mean the package creators recommend using that along with their package, not that they are actually using it in their package. Despite these shortcomings, it is estimated that presence of a src directory or the use of specific packages is an indication that some optimization effort was put into a package.

As shown in Figure 3, the evaluation for the presence of a non-empty src directory shows that there is an increasing trend in using compiled code in R packages, by count. However, when evaluated as a percent of all $\mathrm{R}$ packages, the change has only been a slight increase over the last few years. Table S3 contains the data used to generate Figure 3. 
199

200

201

202

203

204

205

206

207

208

209

210

211

212

213

214

215

216

217

218

219

220

221

222

223

224

225

226

227

228

229

230

231

232

233

234

235

236

237

238

As shown in Figure 4, in 2018, Rcpp is the most common optimization related dependency followed by parallel and foreach. Those same packages have been the most popular for packages last updated during the entire period shown. There 699 packages that list dependencies to more than one optimization framework (407 with 2 dependencies, $220 \mathrm{w} / 3,53 \mathrm{w} / 4,16 \mathrm{w} / 5,2$ $\mathrm{w} / 6,1 \mathrm{w} / 7)$, so the total number of packages shown in the histogram includes some that are double-counted. Table S4 contains the data used to generate Figure 4.

As the numbers from Metric 1 do not match the numbers of Metric 2, some additional exploration is necessary. In terms of total difference, there are 818 more packages using compiled code vs those with one of the searched for dependencies. There are 1726 packages that do not list a dependency to one of the specified packages, but have a src directory for compiled code. There are 908 packages that list a dependency to one of the specified packages but do not have a src directory. See Supplemental tables S3 and S4 for more details.

\section{RECOMMENDATIONS TO IMPROVE QUALITY AND PERFORMANCE}

\section{SOFTWARE TESTING}

Whenever software is written as part of a research project, careful consideration should be given to how to verify that the software performs the desired functionality and produces the desired output. As with bench science, software can often have unexpected and unintended results due to minor or even major problems during the implementation process. Software testing is a wellestablished component of any software development lifecycle (Atchison et al., 1968) and should also be a key component of research software. As shown previously, even among R software packages intended to be shared with and used by others, the majority of R packages $(67 \%$ to $73 \%$ depending on metric) do not have tests that are made available with the package.

Various methodologies and strategies exist for software testing and validation as well as how to integrate software with a software development lifecycle. Some common testing strategies are no strategy, ad hoc testing (Agruss \& Johnson, 2000), test driven development (TDD) (Beck \& Gamma, 1998). There are also common project methodologies where testing fits into the project lifecycle; two common examples are the waterfall project management methodology, where testing is a major phase that occurs at a specific point in time, and the agile project management methodology (Beck et al., 2001), where there are many small iterations including testing. While a full discussion of various methods and strategies is beyond the scope of this article, three key concepts presented are: when to start testing, what to test, and how to test.

\section{Key recommendations for when to test:}

- Build tests before implementation

- Test after functionality has been implemented 
240

241

242

243

244

245

246

247

248

249

250

251

252

253

254

255

256

257

258

259

260

261

262

263

264

265

266

267

268

269

270

271

272

273

274

275

276

277

278

Discussion: One of the popular movements in recent years has been to develop tests first and then implement code to meet desired functionality, a strategy called TDD. While the TDD strategy has done much to improve the focus of the software engineering world on testing, some have found that it does not work with all development styles (Hansson, 2014; Sommerville, 2016), and others have reported that it does not increase developer productivity, reduce overall testing effort, nor improve code quality in comparison to other testing methodologies (Fucci et al., 2016). An approach that more closely matches the theoretically based software development cycle and flexible nature of research software is to create tests after a requirement or feature has been implemented (Osborne et al., 2014; Kanewala \& Bieman, 2014). As developing comprehensive tests of software functionality can be a large burden to accrue at a single point in time, a more pragmatic approach is to alternate between developing new functionality and designing tests to validate new functionality. Similar to the agile software development strategy, a build/test cycle can allow for quick cycles of validated functionality that help to provide input into additional phases of the software lifecycle.

\section{Key recommendations for what to test:}

- Identify the most important or unique feature(s) of software being implemented. Software bugs are found to follow a Pareto or Zipfian distribution.

- Test data and software configuration

- If performance is a key feature, build tests to evaluate performance.

Discussion: In an ideal world, any software developed would be accompanied by $100 \%$ test coverage validating all lines of code, all aspects of functionality, all input, and all interaction with other software. However, due to pressures of research, having time to build a perfect test suite is not realistic. A parsimonious application of the Pareto principle will go a long way towards improving overall software quality without adding to the testing burden. Large companies such as Microsoft have applied traditional scientific methods to the study of bugs and found that the Pareto principle matches reality: $20 \%$ of bugs cause $80 \%$ of problems; additionally a Zipfian distribution may apply as well: $1 \%$ of bugs cause $50 \%$ of all problems (Rooney, 2002).

To apply the Pareto principle to testing, spend some time in a thought experiment to determine answers to questions such as: What is the most important feature(s) of this software? If this software breaks, what is the most likely bad outcome? For computationally intensive components - how long should this take to run?

Once answers to these questions are known, the developer(s) should spend time designing tests to validate key features, avoiding major negatives, and ensuring software performs adequately. Optimization and performance recommendations are covered in the "Software Optimization" section. Part of the test design process should include how to "test" more than just the code. 
279 Some specific aspects of non-code tests include validation of approach and implementation 280 choices with a mentor or colleague.

281

282 As a brief example of how to apply the aforementioned testing principles, we provide some

283 information on testing steps followed during the pccc package development process. The first 284 tests written were those that were manually developed and manually run as development

285 progressed. Key test cases of this form are ideal candidates for inclusion in automated testing. 286 The first tests were taking a known data set, running our process to identify how many of the 287 input rows had complex chronic conditions, and then report on the total percentages found; this 288 result was then compared with published values.

289

290

291

292

293

294

295

296

297

298

299

300

301

302

303

304

305

306

307

308

309

310

311

312

313

314

315

316

317

318

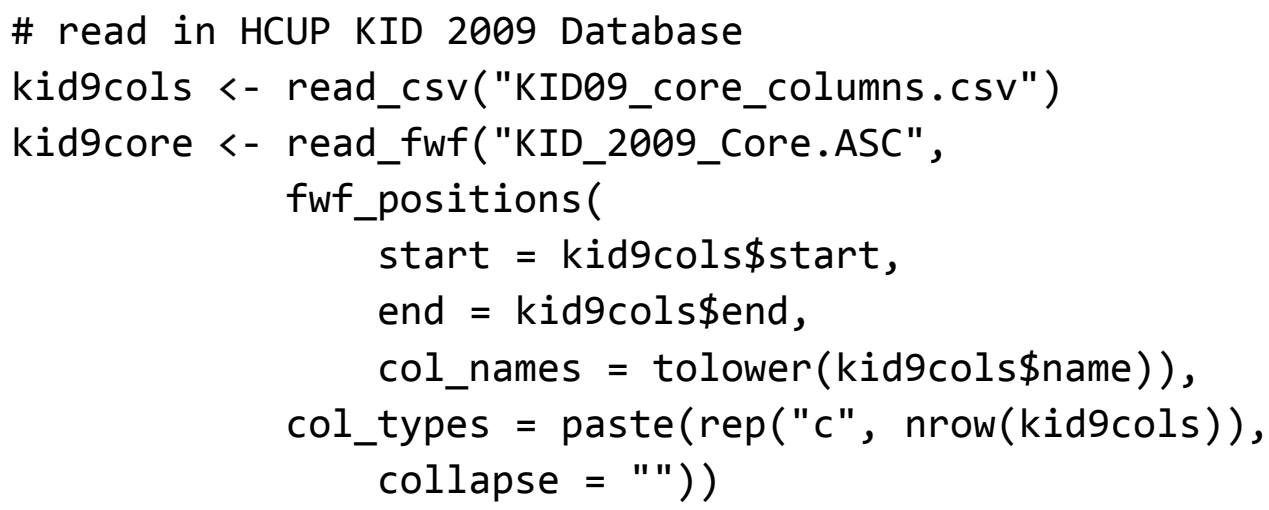


321

322

323

324

325

326

327

328

329

330

331

332

333

334

335

336

337

338

339

340

341

342

343

344

345

346

347

348

349

350

351

352

353

354

355

356

357

358

$$
\begin{aligned}
& \text { dplyr:: summarize_at(kid_ccc, vars(-recnum), mean) } \\
& \%>\% \text { print.data.frame }
\end{aligned}
$$

For the pccc package there is a large set of ICD codes and code set patterns that are used to determine if an input record meets any complex chronic condition criteria. To validate the correct functioning of the software, we needed to validate the ICD code groupings were correct and were mutually exclusive (as appropriate). As pccc is a re-implementation of existing SAS and Stata code, we needed to validate that the codes from the previously developed and published software applications were identical and were performing as expected. Through a combination of manual review and automated comparison codes were checked to see if duplicates and overlaps existed. Any software dealing with input validation or having a large amount of built-in values used for key functionality should follow a similar data validation process.

As an example of configuration testing, here is a brief snippet of some of the code used to automatically find duplicates and codes that were already included as part of another code:

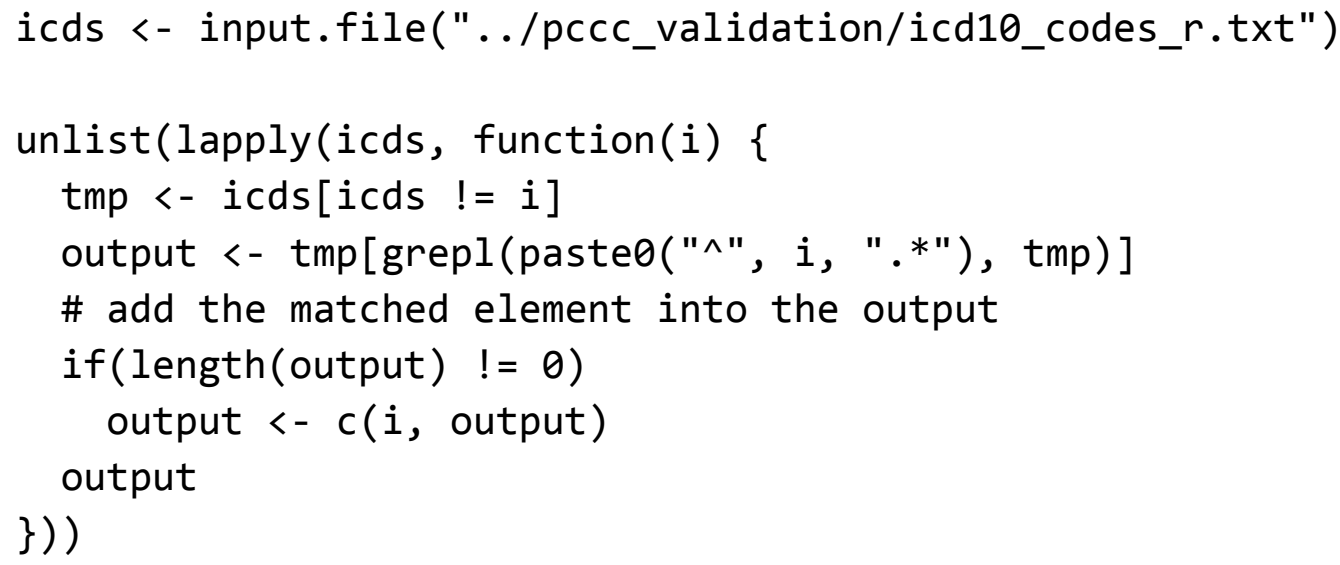

\section{Key recommendations for how to test:}

- Software developer develops unit tests

- Intended user of software should perform validation/acceptance tests

- Run all tests regularly

- Review key algorithms with domain experts.

Discussion: Most programming languages have a multitude of testing tools and frameworks available for assisting developers with the process of testing software. Due to the recurring patterns common across programming languages most languages have a SUnit (Wikipedia contributors, 2017a) derived testing tool, commonly referred to as an "xUnit" (Wikipedia contributors, 2017b) testing framework that focuses on validating individual units of code along 
with necessary input and output meet desired requirements. Based on software language used,

360

361

362

363

364

365

366

367

368

369

370

371

372

373

374

375

376

377

378

379

380

381

382

383

384

385

386

387

388

389

390

391

392

393

394

395

396

397

398

unit tests may be at the class or function/procedure level. Some common xUnit style packages in $\mathrm{R}$ are RUnit and testthat. Unit tests should be automated and run regularly to ensure errors are caught and addressed quickly. For R, it is easy to integrate unit tests into the package build process, but other approaches such as post-commit hook in a version control system are also common.

In addition to unit tests, typically written by the developers of the software, users should perform acceptance tests, or high-level functionality tests that validate the software meets requirements. Due to the high-level nature and subjective focus of acceptance tests, they are often manually performed and may not follow a regimented series of steps. Careful documentation of how a user will actually use software, referred to as user stories, are translated into step by step tests that a human follows to validate the software works as expected. A few examples of acceptance testing tools that primarily focus GUI aspects of software are: Selenium (Selenium Contributors, 2018), Microfocus Unified Functional Testing (formely known as HP's QuickTest Professional) (Micro Focus, 2018), and Ranorex (Ranorex $\mathrm{GmbH}, 2018$ ). As research focused software often does not have a GUI, one aide to manual testing processes is for developers of the software or expert users to create a full step by step example via an R Markdown (Allaire et al., 2018b; Xie, Allaire \& Grolemund, 2018) notebook demonstrating use of the software followed by either manually or automatic validation that the expected end result is correct.

In addition to the tool-based approaches already mentioned, other harder to test items such as algorithms and solution approach should be scrutinized as well. While automated tests can validate mathematical operations or other logic steps are correct, they cannot verify that the approach or assumptions implied through software operations are correct. This level of testing can be done through code review and design review sessions with others who have knowledge of the domain or a related domain.

During development of the pccc package, after the initial tests shown in previous sections, further thought went into how the specifics of the desired functionality should perform. Unit tests were developed to validate core functionality. We also spent time thinking about how the software might behave if the input data was incorrect or if parameters were not specified correctly. If an issue is discovered at this point, a common pattern is to create a test case for discovered bugs that are fixed - this ensures that a re-occurrence, known as a "regression" to software engineers, of this error does not happen again. In the case of pccc, developers expected large input comprised of many observations with many variables. When a tester accidentally just passed 1 observation with many variables, the program crashed. The problem was discovered to be due to the flexible nature of the sapply () function returning different data types based on input. 
399 The original code from ccc.R:

400

401

402

403

404

405

406

407

408

409

410

411

412

413

414

415

416

417

418

419

420

421

422

423

424

425

426

427

428

429

430

431

432

433

434

435

436

437

438

\# check if call didn't specify specific diagnosis columns

if (!missing(dx_cols)) \{

\# assume columns are referenced by ' $d x$ ccols'

dxmat <- sapply(dplyr: : select (

$$
\text { data, !!dplyr::enquo(dx_cols)), as.character) }
$$

\# create empty matrix if necessary

if(! is.matrix(dxmat)) \{

dxmat <- as.matrix(dxmat)

\}

\} else \{

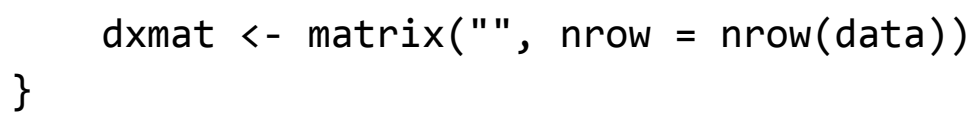

The new code:

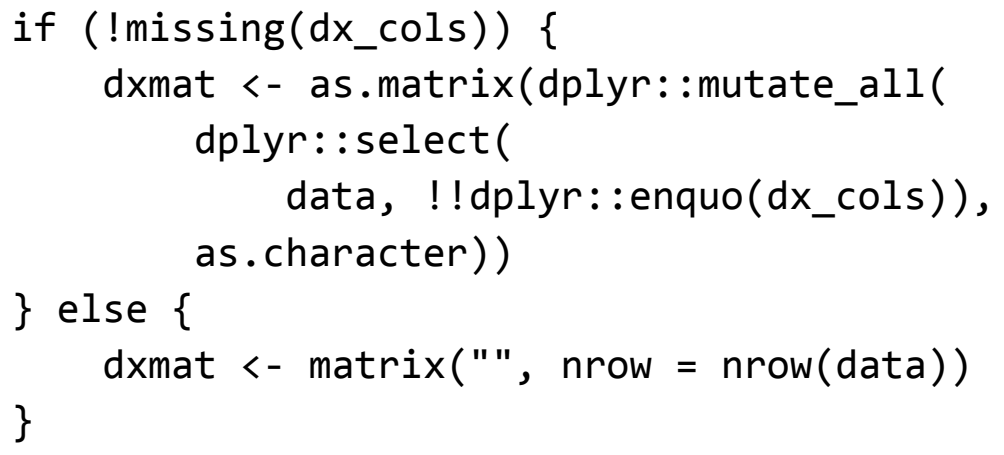

One of the tests written to verify the problem didn't reoccur:

\# Due to previous use of sapply in ccc.R, this would fail test_that(paste(" 1 patient with multiple rows of no diagnosis", "data - should have all CCCS as FALSE"), \{ expect_true(all(ccc(dplyr::data_frame)(

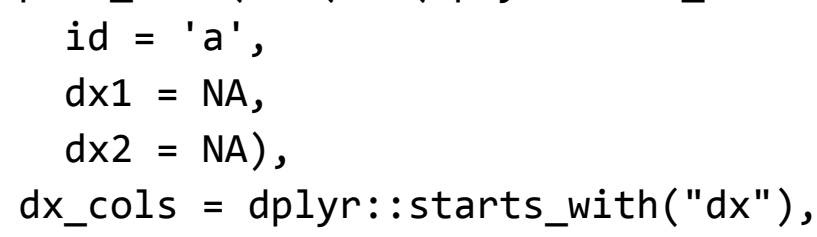


440

441

442

443

444

445

446

447

448

449

450

451

452

453

454

455

456

457

458

459

460

461

462

463

464

465

466

467

468

469

470

471

472

473

474

475

476

477

Testing Anti-Patterns: While the above guidance should help researchers know the basics of testing, it does not cover in detail what not to do. An excellent collection of testing anti-patterns can be found at (Moilanen, 2014; Carr, 2015; Stack Overflow Contributors, 2017). Some key problems that novices experience when learning how to test software are:

- Interdependent tests - Interdependent tests can cause multiple test failures. When a failure in an early test case breaks a later test, it can cause difficulty in resolution and remediation.

- Testing application performance - While testing execution timing or software performance is a good idea and is covered more in the "Software Optimization" section, creating an automated test to perform this is difficult and does not carry over well from one machine to another.

- Slow running tests - As much as possible, tests should be automated but still run quickly. If the testing process takes too long consider refactoring tests or evaluating the performance of the software being tested.

- Only test correct input - A common problem in testing is to only validate expected inputs and desired behavior. Make sure tests cover invalid input, exceptions, and similar items.

\section{SOFTWARE OPTIMIZATION}

Getting software to run in a reasonable amount of time is always a key consideration when working with large datasets. A mathematical understanding of software algorithms is usually a key component of software engineering curricula, but not widely covered in other disciplines. Additionally, while software engineering texts and curricula highlight the importance of testing for non-functional requirements such as performance (Sommerville, 2015), they often fail to provide details on how best to evaluate software performance or how to plan for performance during the various phases of software lifecycle.

The survey of R packages at the beginning of this work indicates that approximately $75 \%$ of packages do not use optimization related packages nor compiled code to improve performance. While the survey of R packages is not evidence of non-optimization of packages in CRAN, computational researchers can should carefully consider performance aspects of their software before declaring it complete. This section will provide a starting point for additional study, research, and experimentation. The Python Foundation's Python language wiki provides excellent high-level advice (Python Wiki Contributors, 2018) to follow before spending too much time in optimization: First get the software working correctly, test to see if it is correct, profile the application if it is slow, and lastly optimize based on the results of code profiling. If necessary, repeat multiple cycles of testing, profiling, and optimization phases. The key aspects 
478 of software optimization discussed in this are: identify a performance target, understanding and

479 applying Big $\mathrm{O}$ notation, and the use code profiling and benchmarking tools.

480

481

482

483

484

485

486

487

\section{Key recommendations for identifying and validating performance targets:}

- Identify functional and non-functional requirements of the software being developed.

- If software performance is key to the software requirements, develop repeatable tests to evaluate performance

488

489

490

491

492

493

494

495

496

497

498

499

500

501

502

503

504

505

506

507

508

Discussion: The first step to software optimization is to understand the functional and nonfunctional requirements of the software being built. Based on expected input, output, and platform the software will be run on, one can make a decision as to what is good enough for the software being developed. A pragmatic approach is best - do not spend time optimizing if it does not add value. Once the functional requirements have been correctly implemented and validated, a decision point is reached: decide if the software is slow and in need of evaluation and optimization. While this may seem a trivial and unnecessary step, it should not be overlooked; a careful evaluation of costs versus benefit from an optimization effort should be evaluated before moving forward. Some methods for gathering the performance target are through an evaluation of other similar software, interdependencies of the software and its interaction with other systems, and discussion with other experts in the field.

Once a performance target has been identified, development of tests for performance can begin. While performance testing is often considered an anti-pattern of testing (Moilanen, 2014) some repeatable tests should be created to track performance as development progresses. Often a 'stress test' or a test with greater than expected input/usage is the best way to do this. A good target is to check an order of magnitude larger input than expected. This type of testing can provide valuable insight into the performance characteristics of the software as well unearth potentials for failure due to unexpected load (Sommerville, 2015).

Here is an example of performance validation testing that can also serve as a basic reproducibility test calling the main function from pccc using the microbenchmark package (one could also use bench, benchr, or other similar R packages).

509

510

511

512

513

514

515

516

517

518

library (pccc)

$$
\begin{aligned}
& \mathrm{rm}(\operatorname{list}=\operatorname{ls}()) \\
& \mathrm{gc}()
\end{aligned}
$$

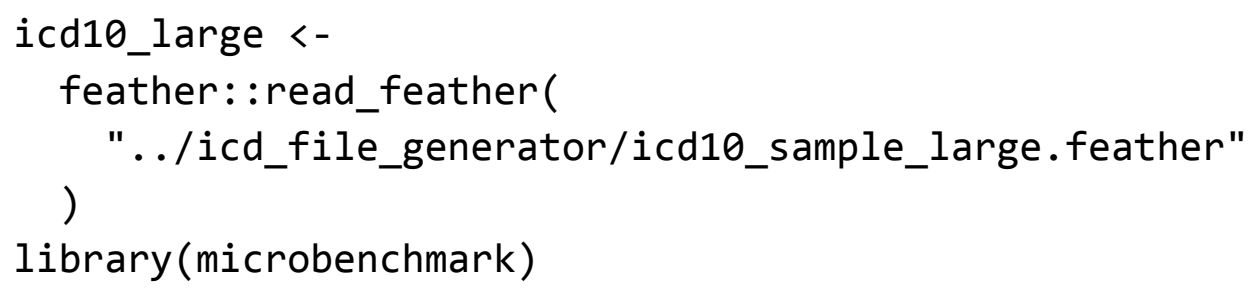


519

520

521

522

523

524

525

526

527

528

529

530

531

532

533

534

535

536

537

538

539

540

541

542

543

544

545

546

547

548

549

550

551

552

553

554

555

556

557

558

microbenchmark( ccc(icd10_large[1:10000, c(1:45)], \# get id, dx, and pc columns id $\quad=i d$, $d x \_c o l s=$ dplyr::starts_with $(" d x ")$, pc_cols = dplyr::starts_with $(" p c ")$, icdv $=10$ ),

times $=10)$

Unit: seconds

expr min $1 q$ mean median uq max neval

$\begin{array}{llllllll}\text { CCC } & 2.857625 & 2.908964 & 2.959805 & 2.920408 & 3.023602 & 3.119937 & 10\end{array}$

Results are from a system with 3.1 GHz Intel Core i7, 16 GB 2133 MHz LPDDR3, PCI-Express SSD, running macOS 10.12.6 and $\mathrm{R}$ version 3.5.1 (2018-07-02).

As software runs can differ significantly from one to the next due to other software running on the test system, a good starting point is to run the same test 10 times (rather than the microbenchmark default of 100 due to this being a longer running process) and record the mean run time. microbenchmark also shows median, lower and upper quartiles, min, and max run times. The actual $\operatorname{ccc}()$ call specifics are un-important; the key is to test the main features of your software in a repeatable fashion and watch for performance changes over time. These metrics can help to identify if a test was valid and indicate a need for retesting; i.e. a large interquartile range may indicate not enough tests were run or some aspect of environment is causing performance variations. Software benchmarking is highly system specific in that changing OS version, $\mathrm{R}$ version, $\mathrm{R}$ dependent package version, compiler version (if compiled code involved), or hardware may change the results. As long as all tests are run the same on the same system with the same software, one can compare timings as development progresses.

Lastly, although the example above is focused on runtime, it can be beneficial to also identify targets for disk space used and memory required to complete all desired tasks. As an example, tools such as bench and profvis demonstrated in our 'Code Profiling/Benchmarking' section as well as object.size() from core R can give developers insight into memory allocation and usage. There are many resources beyond this work that can provide guidance on how to minimize RAM and disk resources (Kane, Emerson \& Weston, 2013; Wickham, 2014b; Wickham et al., 2016; Klik, Collet \& Facebook, 2018).

\section{Key recommendations for identifying upper bound on performance:}

- Big O notation allows the comparison of theoretical performance of different algorithms

- Evaluate how many times blocks of code will run as input approaches infinity

- Loops inside loops are very slow as input approaches infinity 
560 Discussion: Big O notation is a method for mathematically determining the upper bound on 561 performance of a block of code without consideration for language and hardware specifics.

562 Although performance can be evaluated in terms of storage or run time, most examples and 563 comparisons focus on run time. However, when working with large datasets, memory usage and 564 disk usage can be of equal or higher importance than run. Big O notation is reported in terms of 565 input (usually denoted as n) and allows one to quickly compare theoretical performance of 566 different algorithms.

567

The basic steps for evaluating the upper bound of performance of a block of software code is to

569 evaluate what code will run as $n$ approaches infinity. Items that are constant time (regardless of if they run once or $\mathrm{x}$ times independent of input) are reduced down to $\mathrm{O}(1)$. The key factors that

571 contribute to Big O are loops - a single for loop or similar construct through recursion that runs once for all $\mathrm{n}$ is $\mathrm{O}(\mathrm{n})$; a nested for loop would be $\mathrm{O}\left(\mathrm{n}^{2}\right)$. When calculating Big $\mathrm{O}$ for a code block, function, or software system, lower order terms are ignored, and just the largest Big $\mathrm{O}$ notation is used; for example if a code block is $\mathrm{O}(1)+\mathrm{O}(\mathrm{n})+\mathrm{O}\left(\mathrm{n}^{3}\right)$ it would be denoted as

$575 \mathrm{O}\left(\mathrm{n}^{3}\right)$.

576

577

578

579

580

581

582

583

584

585

586

587

588

589

590

591

592

593

594

595

596

597

Despite the value of understanding the theoretical upper bound of software in an ideal situation, there are many difficulties that arise during implementation that can make Big O difficult to calculate and which could make a large Big $\mathrm{O}$ faster than a small Big $\mathrm{O}$ under actual input conditions. Some key takeaways to temper a mathematical evaluation of $\mathrm{Big} \mathrm{O}$ are:

- Constants matter when choosing an algorithm - for example if one algorithm is $\mathrm{O}\left(56 \mathrm{n}^{2}\right)$, there exists some $\mathrm{n}$ where $\mathrm{O}\left(\mathrm{n}^{3}\right)$ is faster.

- Average or best case run time might be more relevant.

- Big O evaluation of algorithms in high level languages is often hard to quantify.

For additional details on Big $\mathrm{O}$ notation, see the excellent and broadly understandable introduction to Big O notation (Abrahms, 2016).

\section{Key recommendations for profiling and benchmarking:}

- Profile code to find bottlenecks

- Modify code to address largest items from profiling

- Run tests to make sure functionality isn't affected

- Repeat process if gains are made and additional performance improvements are necessary.

598

Discussion: As discussed throughout this section, optimization is a key aspect of software development, especially with respect to large datasets. Although identification of performance 
599 targets and a mathematical analysis of algorithms are important steps, the final result must be 600 tested and verified. The only way to know if your software will perform adequately under ideal 601 (and non-ideal) circumstances is to use benchmarking and code profiling tools. Code profilers 602 show how a software behaves and what functions are being called while benchmarking tools 603 generally focus on just execution time - though some tools combine both profiling and 604 benchmarking. In R, some of the common tools are bench, benchr, microbenchmark, 605 tictoc, Rprof (R Core Team, 2018), proftools (Tierney \& Jarjour, 2016), and profvis.

606

607 If, after implementation has been completed, the software functions correctly, and performance 608 targets have not been met, look to optimize your code. Follow an iterative process of profiling to 609 find bottlenecks, making software adjustments, testing small sections with benchmarking and 610 then repeating the process with overall profiling again. If at any point in the process you discover 611 that due to input size, functional requirements, hardware limitations, or software dependencies 612 you cannot make a significant impact to performance, consider stopping further optimization 613 efforts (Burns, 2012).

614

As with software testing and software bugs, the Pareto principle applies, though some put the balance between code and execution time is closer to $90 \%$ of time is in $10 \%$ of the code or even as high as $99 \%$ in 1\% (Xochellis, 2010; Bird, 2013). Identify the biggest bottlenecks via code

618 profiling and focus only on the top issues first. As an example of how to perform code profiling and benchmarking in $\mathrm{R}$, do the following:

620

621

622

623

624

625

626

627

628

629

630

631

632

633

634

635

636

637

First, use profvis to identify the location with the largest execution time:

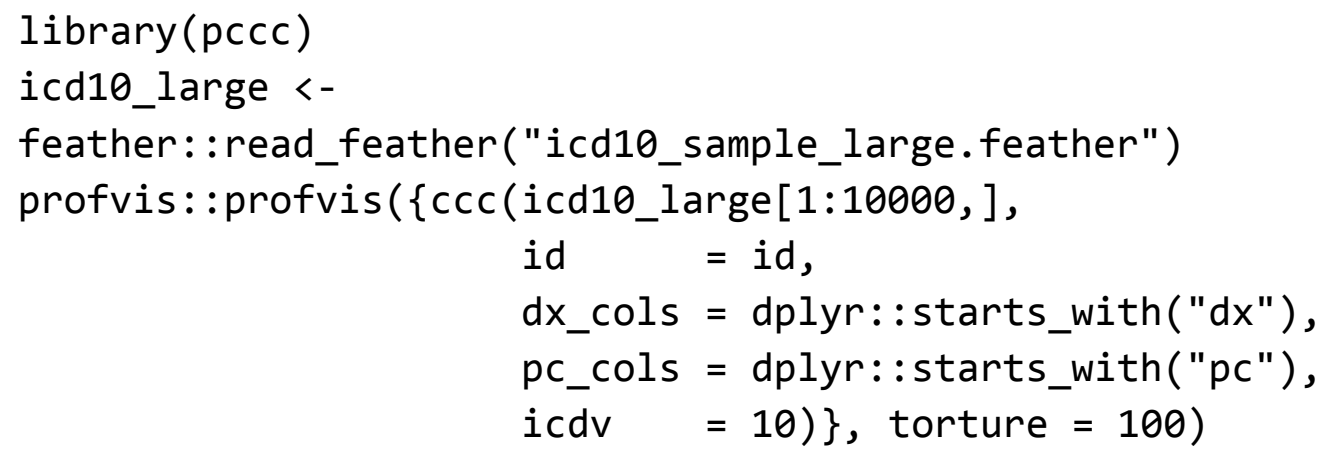

In Figure 5 you can see a visual depiction of memory allocation, known as a "Flame Graph", as well as execution time and call stack. By clicking on each item in the stack you will be taken directly to the relevant source code and can see which portions of the code take the most time or memory allocations. Figure 6 is a depiction of the data view which shows just the memory changes, execution time, and source file. 
638 Once the bottleneck has been identified, if possible extract that code to a single function or line 639 that can be run repeatedly with a library such as microbenchmark or tictoc to see if a small

640 change either improves or degrades performance. Test frequently and make sure to compare

641 against previous versions. You may find that something you thought would improve

642 performance degrades performance. As a first step we recommend running tictoc to get

643 general timings such as the following:

644

645

646

647

648

649

650

651

652

653

654

655

656

657

658

659

660

661

662

663

664

665

library(tictoc)

tic("timing: $r$ version")

out <- dplyr::bind_cols(ids, ccc_mat_r(dxmat, pcmat, icdv))

toc ()

666

667

668

669

670

671

672

673

674

675

tic("timing: c++ version") dplyr: :bind_cols(ids, ccc_mat_rcpp(dxmat, pcmat, icdv)) toc ( )

timing: $r$ version: $37.089 \mathrm{sec}$ elapsed

timing: c++ version: $5.087 \mathrm{sec}$ elapsed

As with previous timings, while we're showing pccc calls, any custom function of block of code you have can be compared against an alternative version to see which performs better. The above blocks of code call the core functionality of the pccc package - one implemented all in R, the other with $\mathrm{C}++$ for the matrix processing and string matching components; see sourcecode available at https://github.com/magic-lantern/pccc/blob/no_cpp/R/ccc.R for full listing.

After starting with high level timings, next run benchmarks on specific sections of code such as in this example comparing importing a package vs using the package reference operator using bench:

676

677

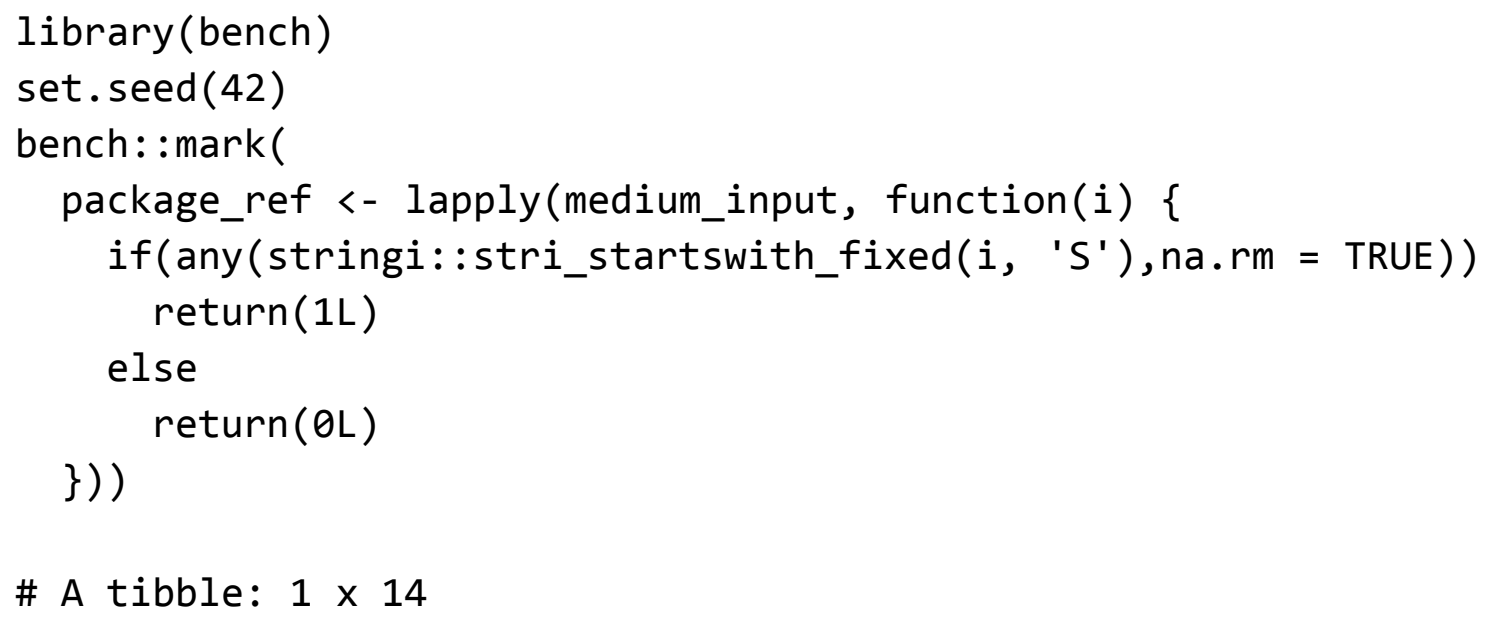


678

679

680

681

682

683

684

685

686

687

688

689

690

691

692

693

694

695

696

697

698

699

700

701

702

703

704

705

706

707

708

709

710

711

712

713

714

715

716

717 Some general lessons learned from profiling and benchmarking: $80 \%$. our source code repository for some guidance on this topic.

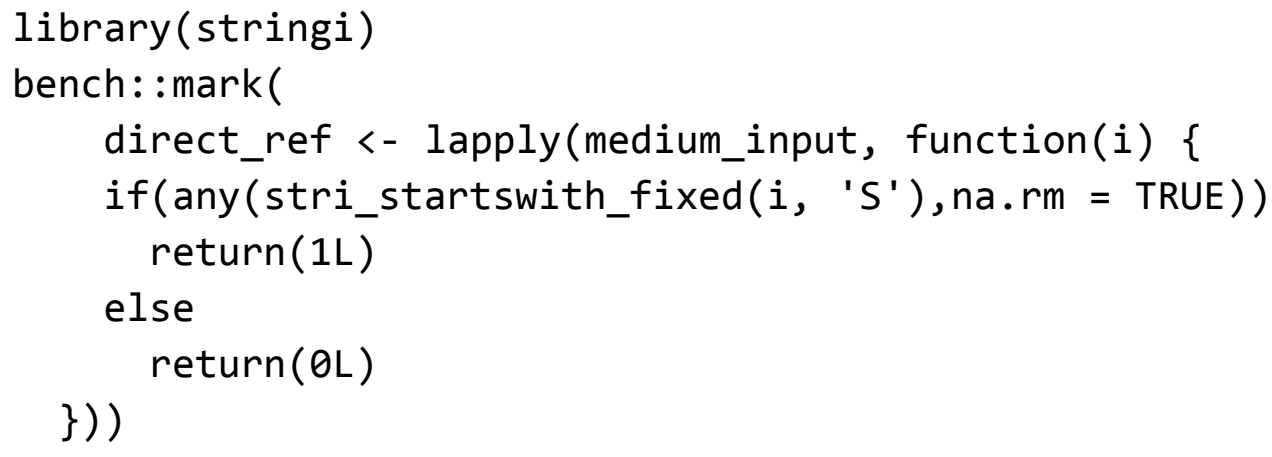

The above test was run on a virtual machine running Ubuntu 16.04.5 LTS using R 3.4.4.

One benefit of bench: : mark over microbenchmark is that bench reports memory allocations as well as timings, similar to data shown in profvis. Through benchmarking we found that for some systems/configurations the use of the "::" operator, as opposed to importing a package, worsened performance noticeably. Also widely known (Gillespie \& Lovelace, 2017) and found to be applicable here is that the use of matrices are preferred for performance reasons over data.frames or tibbles. Matrices do have different functionality, which can require some re-work when converting from one to another. For example, a matrix can only contain 1 data type such as character or numeric; data.frames and tibbles support shortcut notations such as mydf $\$$ colname. Another key point found is that an "env" with no parent environment is significantly faster (up to 50x) than one with a parent env. In the end, optimization efforts resulted in reducing run time by

One limitation with $\mathrm{R}$ profiling tools is that if the code to be profiled executes $\mathrm{C}++$ code, you will get no visibility into what is happening once the switch from $\mathrm{R}$ to $\mathrm{C}++$ has occurred. As shown in Figure 7, visibility into timing and memory allocation stops at the . Call() function. In order to profile $\mathrm{C}++$ code, you need to use non- $\mathrm{R}$ specific tools such as XCode on macOS or gprof on non-macOS Unix-based operating system (OS). See "R_with_C++_profiling.md" in 
- "Beware the dangers of premature optimization of your code. Your first duty is to create clear, correct code." (Knuth, 1974; Burns, 2012) Never optimize before you actually

721

722 know what is taking all the time/memory/space with your software. Different compilers

723 and core language updates often will change or reverse what experience has previously indicated as sources of slowness. Always benchmark and profile before making a change.

\section{CONCLUSION}

741 Researchers frequently develop software to automate tasks and speed the pace of research.

742 Unfortunately, researchers are rarely trained in software engineering principles necessary to

743

744 develop robust, validated, and performant software. Software maintenance is an often overlooked and underestimated aspect in the lifecycle of any software product. Software engineering principles and tooling place special focus on the processes around designing, building, and maintaining software. In this paper, the key topics of software testing and software optimization

747 have been discussed along with some analysis of existing software packages in the R language.

748 Our analysis showed that the majority of $R$ packages have neither unit testing nor evidence of

749 optimization available with normally distributed source code. Through self-education on unit

750 testing and optimization, any computational or other researcher can pick up the key principles of software engineering that will enable them to spend less time troubleshooting software and more

752

753 time doing the research they enjoy.

755

\section{REFERENCES}

Abrahms J. 2016.Big-O notation explained by a self-taught programmer. Available at https://justin.abrah.ms/computer-science/big-o-notation-explained.html 
757 Agruss C, Johnson B. 2000. Ad Hoc Software Testing. Viitattu 4:2009.

758 Allaire JJ, Francois R, Ushey K, Vandenbrouck G, library MG (TinyThread,

759 http://tinythreadpp.bitsnbites.eu/), RStudio, library I (Intel T,

760

761 https://www.threadingbuildingblocks.org/), Microsoft. 2018a. RcppParallel: Parallel

762 Programming Tools for "Rcpp."

763

764

765

766

767

768

769

770

771

772

773

774

775

776

777

778

779

780

781

782

783

784

785

786

Allaire JJ, Xie Y, McPherson J, Luraschi J, Ushey K, Atkins A, Wickham H, Cheng J, Chang W, Iannone R. 2018b. rmarkdown: Dynamic Documents for $R$.

Apache Software Foundation. 2018.SparkR (R on Spark) - Spark 2.3.2 Documentation. Available at https://spark.apache.org/docs/latest/sparkr.html

Atchison WF, Conte SD, Hamblen JW, Hull TE, Keenan TA, Kehl WB, McCluskey EJ, Navarro SO, Rheinboldt WC, Schweppe EJ, Viavant W, Young DM Jr. 1968. Curriculum 68: Recommendations for Academic Programs in Computer Science: A Report of the ACM Curriculum Committee on Computer Science. Commun. ACM 11:151-197. DOI: $10.1145 / 362929.362976$.

Beck K, Beedle M, Bennekum A van, Cockburn A, Cunningham W, Fowler M, Grenning J, Highsmith J, Hunt A, Jeffries R, Kern J, Marick B, Martin RC, Mellor S, Schwaber K, Sutherland J, Thomas D. 2001.Manifesto for Agile Software Development. Available at http://agilemanifesto.org/

Beck K, Gamma E. 1998. Test Infected: Programmers Love Writing Tests. Java Report 3.

Bengtsson H. 2018. future: Unified Parallel and Distributed Processing in R for Everyone.

Bengtsson H, R Core Team. 2018. future.apply: Apply Function to Elements in Parallel using Futures.

Bird J. 2013.Applying the 80:20 Rule in Software Development - DZone Agile. Available at https://dzone.com/articles/applying-8020-rule-software

Bischl B, Lang M. 2015. parallelMap: Unified Interface to Parallelization Back-Ends.

Bischl B, Lang M, Mersmann O, Rahnenführer J, Weihs C. 2015. BatchJobs and BatchExperiments: Abstraction Mechanisms for Using R in Batch Environments. Journal of Statistical Software 64:1-25.

787 Calaway R, Analytics R, Weston S. 2017. doMC: Foreach Parallel Adaptor for "parallel." 
788 Calaway R, Corporation M, Weston S. 2017. doSNOW: Foreach Parallel Adaptor for the 789 "snow" Package.

790 Calaway R, Corporation M, Weston S, Tenenbaum D. 2018. doParallel: Foreach Parallel $791 \quad$ Adaptor for the "parallel" Package.

792 Calaway R, Microsoft, Weston S. 2017. foreach: Provides Foreach Looping Construct for R.

793 Carr J. 2015.TDD Anti-Patterns. Available at

794 https://web.archive.org/web/20150726134212/http://blog.james-

795 carr.org:80/2006/11/03/tdd-anti-patterns/

796 Chang W, Luraschi J. 2018. profvis: Interactive Visualizations for Profiling R Code.

797 Dehaghani SMH, Hajrahimi N. 2013. Which Factors Affect Software Projects Maintenance Cost 798 More? Acta Informatica Medica 21:63-66. DOI: 10.5455/AIM.2012.21.63-66.

799 DeWitt P, Bennett T, Feinstein J, Russell S. 2017. pccc: Pediatric Complex Chronic Conditions. 800 Dragulescu AA, Arendt C. 2018. xlsx: Read, Write, Format Excel 2007 and Excel $80197 / 2000 / X P / 2003$ Files.

802 Eckert A. 2018. parallelDist: Parallel Distance Matrix Computation using Multiple Threads. 803 Eddelbuettel D, Balamuta JJ. 2017. Extending R with $C++$ : A Brief Introduction to Rcpp. PeerJ $804 \quad$ Inc. DOI: $10.7287 /$ peerj.preprints.3188v1.

805 Feinerer I, Theussl S, Buchta C. 2015. DSL: Distributed Storage and List.

806 Feinstein JA, Russell S, DeWitt PE, Feudtner C, Dai D, Bennett TD. 2018. R Package for 807 Pediatric Complex Chronic Condition Classification. JAMA Pediatrics. DOI: $808 \quad$ 10.1001/jamapediatrics.2018.0256.

809 Feudtner C, Christakis DA, Connell FA. 2000. Pediatric Deaths Attributable to Complex Chronic 810 811 Conditions: A Population-Based Study of Washington State, 1980-1997. Pediatrics 106:205-209.

812 Feudtner C, Feinstein JA, Zhong W, Hall M, Dai D. 2014. Pediatric complex chronic conditions 813 814 815 classification system version 2: updated for ICD-10 and complex medical technology dependence and transplantation. BMC Pediatrics 14:199. DOI: 10.1186/1471-2431-14-

816 199.

817

Fucci D, Scanniello G, Romano S, Shepperd M, Sigweni B, Uyaguari F, Turhan B, Juristo N, Oivo M. 2016. An External Replication on the Effects of Test-driven Development Using a Multi-site Blind Analysis Approach. In: Proceedings of the 10th ACM/IEEE 
819

820

821 Gaslam B. 2017. unitizer: Interactive R Unit Tests.

822 Gillespie C, Lovelace R. 2017. Efficient R Programming: A Practical Guide to Smarter

823

824

825

826

827

828

829

830

831

832

833

834

835

836

837

838

839

840

841

842

843

844

845

846

847

848

849
International Symposium on Empirical Software Engineering and Measurement. ESEM '16. New York, NY, USA: ACM, 3:1-3:10. DOI: 10.1145/2961111.2962592.
Glass RL. 2001. Frequently Forgotten Fundamental Facts About Software Engineering. IEEE Softw. 18:112-111. DOI: 10.1109/MS.2001.922739.

Grosjean P. 2014. SciViews-R: A GUI API for R. MONS, Belgium: UMONS.

Hansson DH. 2014.TDD is dead. Long live testing. (DHH). Available at http://david.heinemeierhansson.com/2014/tdd-is-dead-long-live-testing.html

Hester J. 2018. bench: High Precision Timing of R Expressions.

Hyde R. 2009. The Fallacy of Premature Optimization. Ubiquity 2009:1. DOI: 10.1145/1569886.1513451.

Izrailev S. 2014. tictoc: Functions for timing $R$ scripts, as well as implementations of Stack and List structures.

Jones C, Bonsignour O. 2011. The Economics of Software Quality. Addison-Wesley Professional.

Kane M, Emerson J, Weston S. 2013. Scalable Strategies for Computing with Massive Data. Journal of Statistical Software, Articles 55:1-19. DOI: 10.18637/jss.v055.i14.

Kanewala U, Bieman JM. 2014. Testing Scientific Software: A Systematic Literature Review. Information and software technology 56:1219-1232. DOI: 10.1016/j.infsof.2014.05.006.

Kelleher C, Pausch R. 2005. Lowering the Barriers to Programming: A Taxonomy of Programming Environments and Languages for Novice Programmers. ACM Comput. Surv. 37:83-137. DOI: 10.1145/1089733.1089734.

Klevtsov A, Antonov A, Upravitelev P. 2018. benchr: High Precise Measurement of $R$ Expressions Execution Time.

Klik M, Collet Y, Facebook. 2018. fst: Lightning Fast Serialization of Data Frames for $R$.

Knuth DE. 1974. Structured Programming with Go to Statements. ACM Comput. Surv. 6:261301. DOI: $10.1145 / 356635.356640$.

Koskinen J. 2015.Software Maintenance Costs. Available at https://wiki.uef.fi/download/attachments/38669960/SMCOSTS.pdf 
850 Kusnierczyk W, Eddelbuettel D, Hasselman B. 2012. rbenchmark: Benchmarking routine for R. 851 Leek JT, Peng RD. 2015. Opinion: Reproducible research can still be wrong: Adopting a 852 prevention approach. Proceedings of the National Academy of Sciences 112:1645-1646.

853 DOI: $10.1073 /$ pnas.1421412111.

854

855

856

857

858

859

860

861

862

863

864

865

866

867

868

869

870

871

872

873

874

875

876

877

878

879

Lentin J, Hennessey A. 2017. unittest: TAP-Compliant Unit Testing.

Luraschi J, Kuo K, Ushey K, Allaire JJ, Macedo S, RStudio, Foundation TAS. 2018. sparklyr: $R$ Interface to Apache Spark.

Matloff N. 2016. Software Alchemy: Turning Complex Statistical Computations into Embarrassingly-Parallel Ones. Journal of Statistical Software 71:1-15. DOI: 10.18637/jss.v071.i04.

Mersmann O. 2018. microbenchmark: Accurate Timing Functions.

Micro Focus. 2018.Unified Functional Testing. Available at https://software.microfocus.com/enus/products/unified-functional-automated-testing/overview (accessed April 12, 2018).

Moilanen J. 2014.Test Driven Development details. Available at https://github.com/educloudalliance/educloud-development/wiki/Test-DrivenDevelopment-details (accessed April 12, 2018).

Nolan R, Padilla-Parra S. 2017. exampletestr-An easy start to unit testing R packages. Wellcome Open Research 2. DOI: 10.12688/wellcomeopenres.11635.2.

Nutter B, Lane S. 2018. redcapAPI: Accessing data from REDCap projects using the API. DOI: 10.5281/zenodo.11826.

Osborne JM, Bernabeu MO, Bruna M, Calderhead B, Cooper J, Dalchau N, Dunn S-J, Fletcher AG, Freeman R, Groen D, Knapp B, McInerny GJ, Mirams GR, Pitt-Francis J, Sengupta B, Wright DW, Yates CA, Gavaghan DJ, Emmott S, Deane C. 2014. Ten Simple Rules for Effective Computational Research. PLoS Computational Biology 10. DOI: 10.1371/journal.pcbi.1003506.

Prins P, de Ligt J, Tarasov A, Jansen RC, Cuppen E, Bourne PE. 2015. Toward effective software solutions for big biology. Nature Biotechnology 33:686-687. DOI: 10.1038/nbt.3240.

Python Wiki Contributors. 2018.Performance Tips. Available at https://wiki.python.org/moin/PythonSpeed/PerformanceTips (accessed April 12, 2018). 
880 R Core Team. 2018. R: A Language and Environment for Statistical Computing. Vienna, 881 Austria: R Foundation for Statistical Computing.

882 Ranorex GmbH. 2018.Ranorex. Available at https://www.ranorex.com (accessed April 12, 883 2018).

884 Reese J. 2018.Best practices for writing unit tests. Available at https://docs.microsoft.com/en885 us/dotnet/core/testing/unit-testing-best-practices

886

887

Robinson E. 2017.Making R Code Faster : A Case Study. Available at https://robinsones.github.io/Making-R-Code-Faster-A-Case-Study/

888

Rooney P. 2002.Microsoft's CEO: 80-20 Rule Applies To Bugs, Not Just Features. Available at 889

890 http://www.crn.com/news/security/18821726/microsofts-ceo-80-20-rule-applies-to-bugs-

891 not-just-features.htm

892

893

894

895

Sandve GK, Nekrutenko A, Taylor J, Hovig E. 2013. Ten Simple Rules for Reproducible Computational Research. PLOS Computational Biology 9:e1003285. DOI: 10.1371/journal.pcbi.1003285.

Selenium Contributors. 2018.Selenium. Available at https://www.seleniumhq.org (accessed April

896 $12,2018)$.

897

Sommerville I. 2015. Software Engineering. Boston: Pearson.

898

Sommerville I. 2016. Giving up on test-first development. Available at

899 http://iansommerville.com/systems-software-and-technology/giving-up-on-test-first-

900 development/

Stack Overflow Contributors. 2017.Unit testing Anti-patterns catalogue. Available at

901

902 https://stackoverflow.com/questions/333682/unit-testing-anti-patterns-catalogue

903 The Joint Task Force on Computing Curricula. 2015. Curriculum Guidelines for Undergraduate 904 Degree Programs in Software Engineering. New York, NY, USA: ACM.

905 Tierney L, Jarjour R. 2016. proftools: Profile Output Processing Tools for R.

906 Tierney L, Rossini AJ, Li N, Sevcikova H. 2018. snow: Simple Network of Workstations.

907 Weston S. 2017. doMPI: Foreach Parallel Adaptor for the Rmpi Package.

908 Wickham H. 2011. testthat: Get Started with Testing. The R Journal 3:5-10.

909 Wickham H. 2014a. profr: An alternative display for profiling information.

910 Wickham H. 2014b. Advanced R. Boca Raton, FL: Chapman and Hall/CRC. 
911 Wickham H, RStudio, Feather developers, Google, LevelDB Authors. 2016. feather: R Bindings 912 to the Feather "API."

913 Wikipedia contributors. 2017a.SUnit - Wikipedia, The Free Encyclopedia. Available at

914 https://en.wikipedia.org/w/index.php?title $=$ SUnit\&oldid $=815835062$

915 Wikipedia contributors. 2017b.XUnit - Wikipedia, The Free Encyclopedia. Available at

$916 \quad$ https://en.wikipedia.org/w/index.php?title $=X U$ nit\&oldid $=807299841$

917 Wilson G. 2016. Software Carpentry: lessons learned. F1000Research. DOI:

$918 \quad 10.12688 /$ f1000research.3-62.v2.

919 Wilson G, Aruliah DA, Brown CT, Hong NPC, Davis M, Guy RT, Haddock SHD, Huff KD, 920 Mitchell IM, Plumbley MD, Waugh B, White EP, Wilson P. 2014. Best Practices for 921 Scientific Computing. PLOS Biology 12:e1001745. DOI: 10.1371/journal.pbio.1001745.

922 Xie Y. 2018. testit: A Simple Package for Testing R Packages.

923 Xie Y, Allaire JJ, Grolemund G. 2018. R Markdown: The Definitive Guide. Boca Raton, Florida: 924 Chapman and Hall/CRC.

925 Xochellis J. 2010.The impact of the Pareto principle in optimization - CodeProject. Available at 926

927 https://www.codeproject.com/Articles/49023/The-impact-of-the-Pareto-principle-in-

928 Yu H. 2002. Rmpi: Parallel Statistical Computing in R. R News 2:10-14.

929 


\section{Figure 1}

Packages with non-empty testing directory

Count of packages with files in standard testing directories by year a package was last updated. Testing directory "Yes" is determined by the presence of files matching the regular expression "[Tt]est[^/]*/.+"; if no matches are found for an R package, is it counted as a "No."

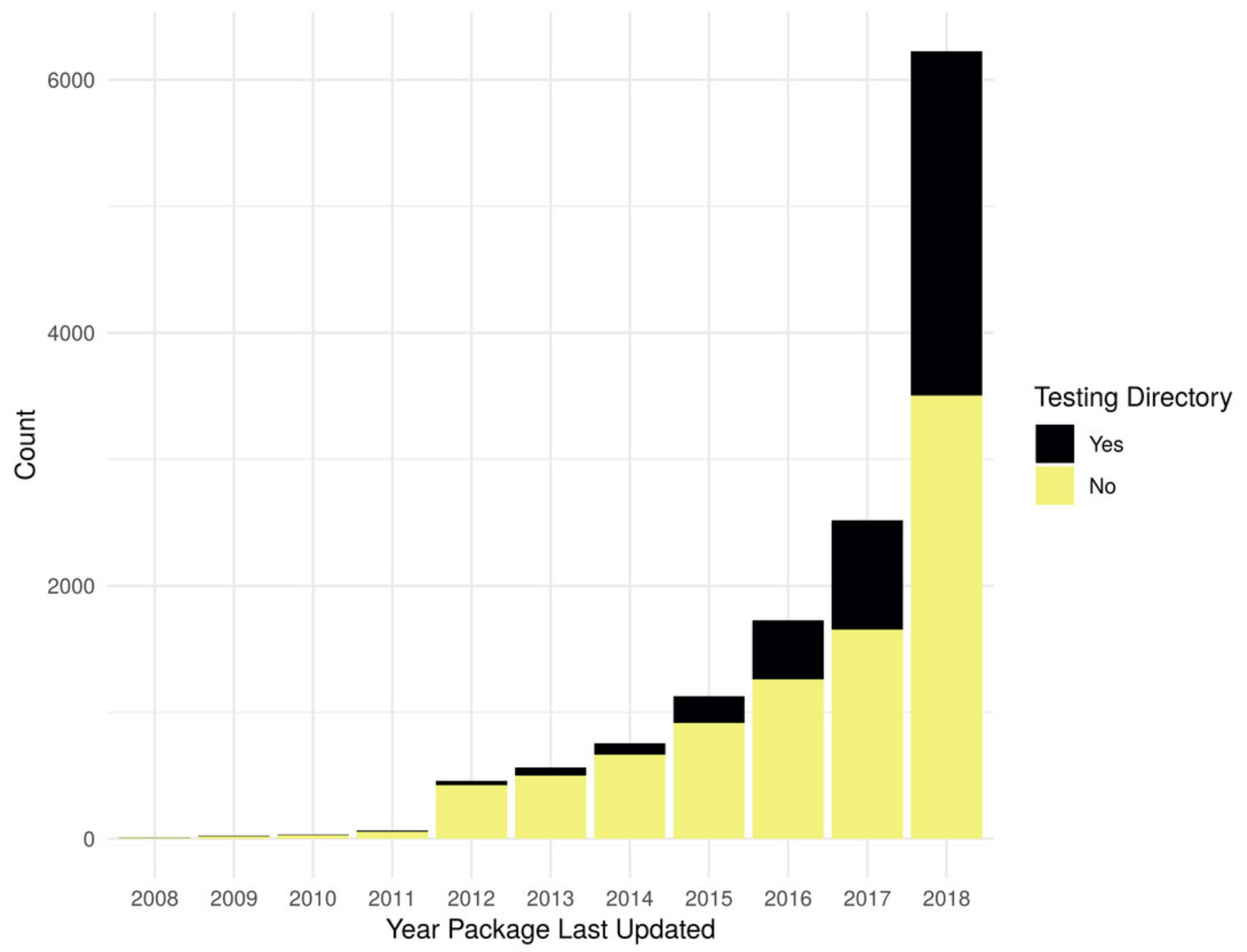


Figure 2

Packages with testing framework dependency

Count of dependencies on a testing package (RUnit, svUnit, testit, testthat, unitizer, unittest) by year a package was last updated. Packages with no stated dependency from their DESCRIPTION file for one of the specified packages are listed as 'none.'

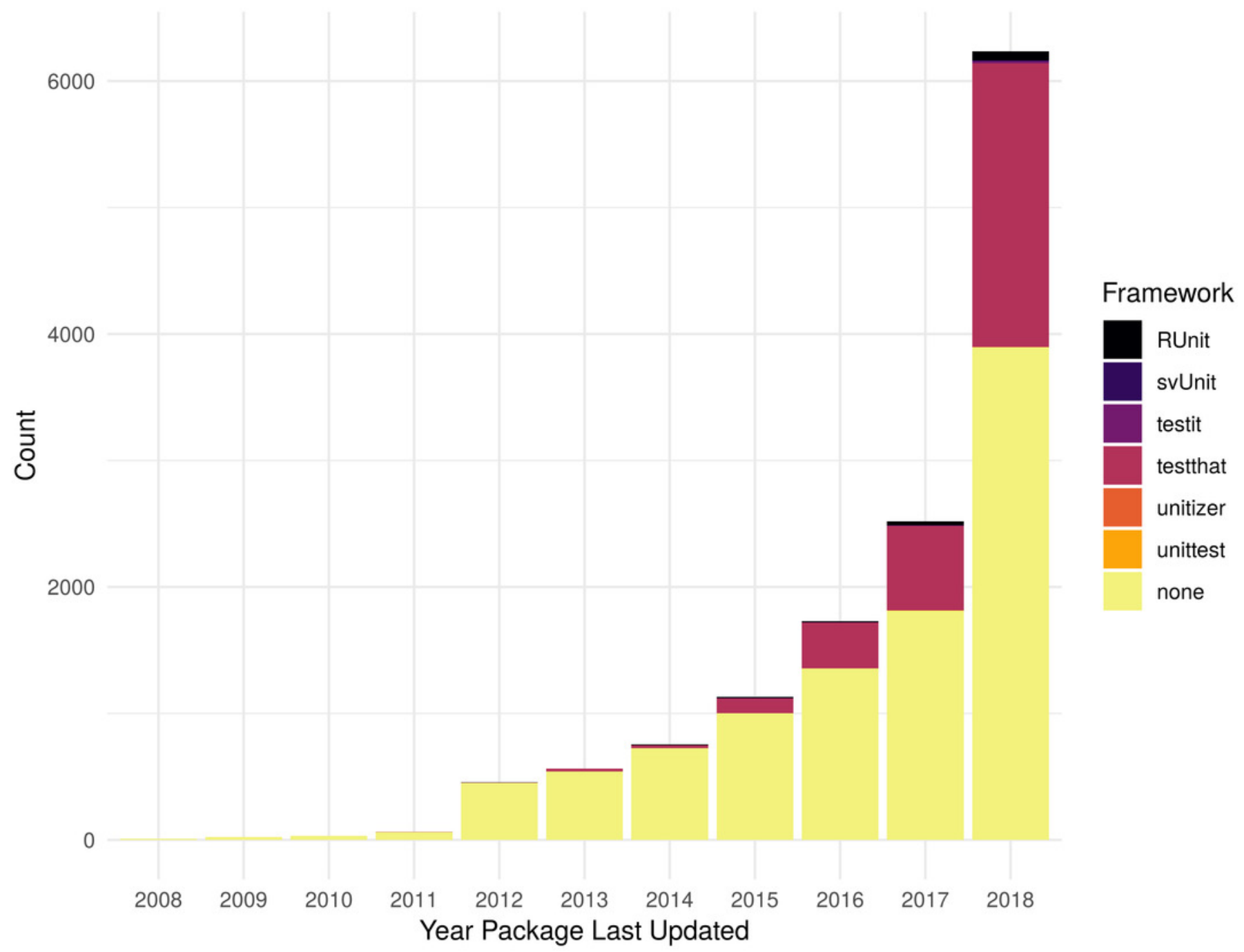




\section{Figure 3}

\section{Packages with non-empty src directory}

Figure 3 Count of packages with files in standard source directories that has code to be compiled by year a package was last updated. Compiled directory "Yes" is determined by the presence of files matching the regular expression "src[^/]*/.+"; if no matches are found for an R package, is it counted as a "No."

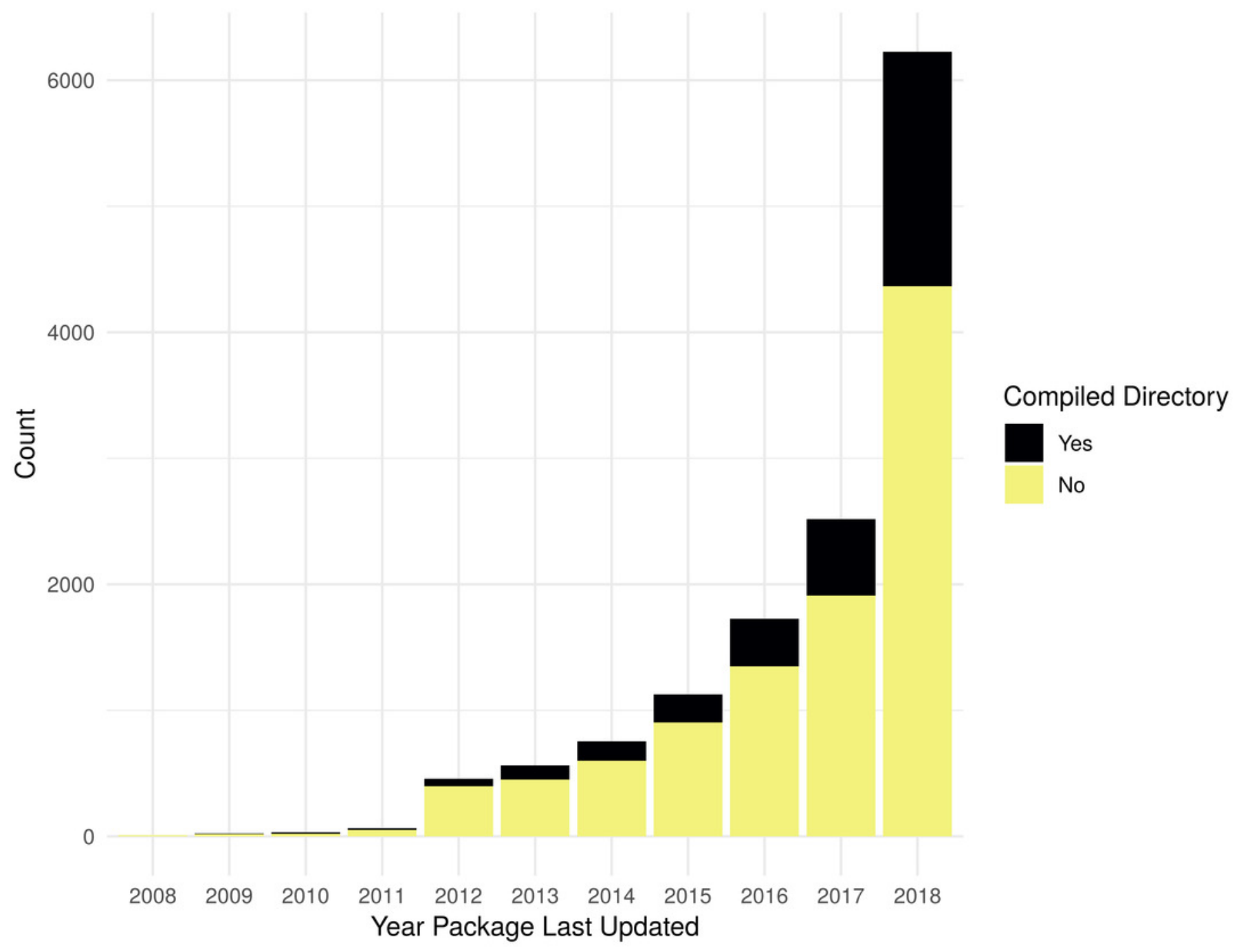




\section{Figure 4}

Packages with optimization framework dependency

Count of dependencies on an optimization related package, see "OPTIMIZATION OF R

PACKAGES" section for complete list, by year a package was last updated. Packages with no stated dependency from their DESCRIPTION file for one of the specified packages are listed as 'none.' In order to aid visual understanding of top dependencies, we limited display to those packages that had 14 or more dependent packages.

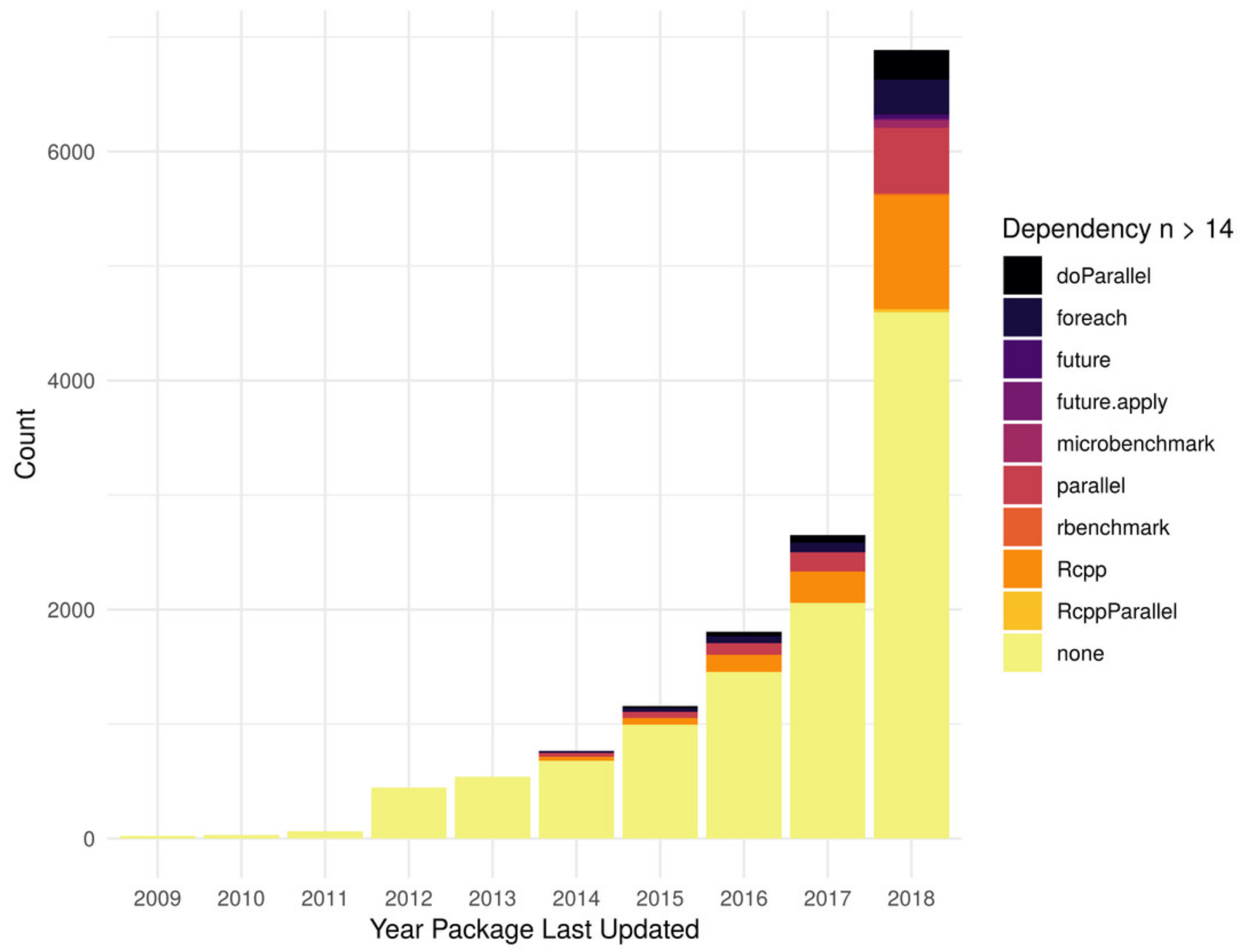


Figure 5

\section{Profvis Flame Graph}

Visual depiction of memory allocation/deallocation, execution time, and call stack.

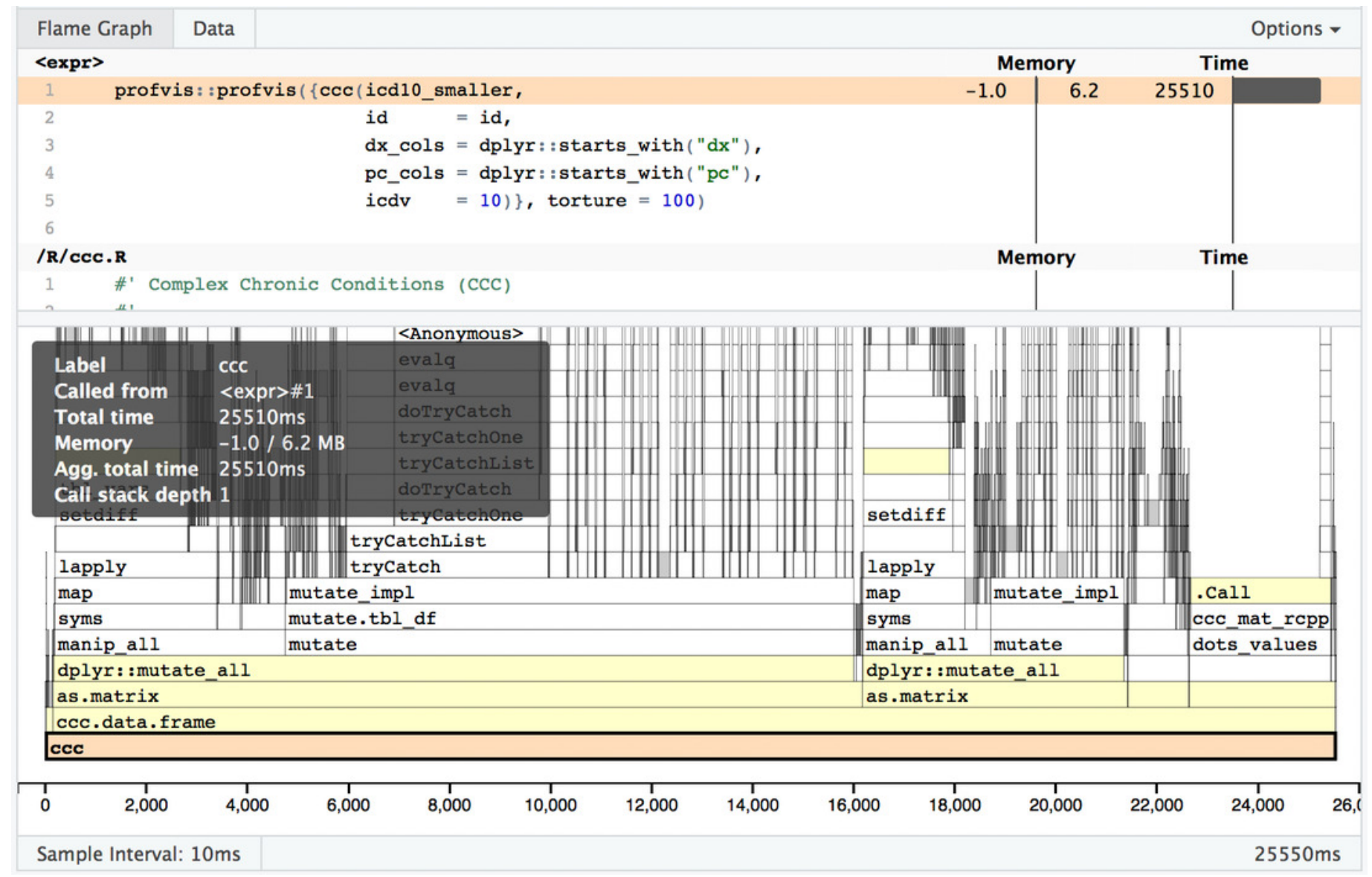




\section{Figure 6}

\section{Profvis Data Chart}

Table view of memory allocation/deallocation, execution time, and call stack.

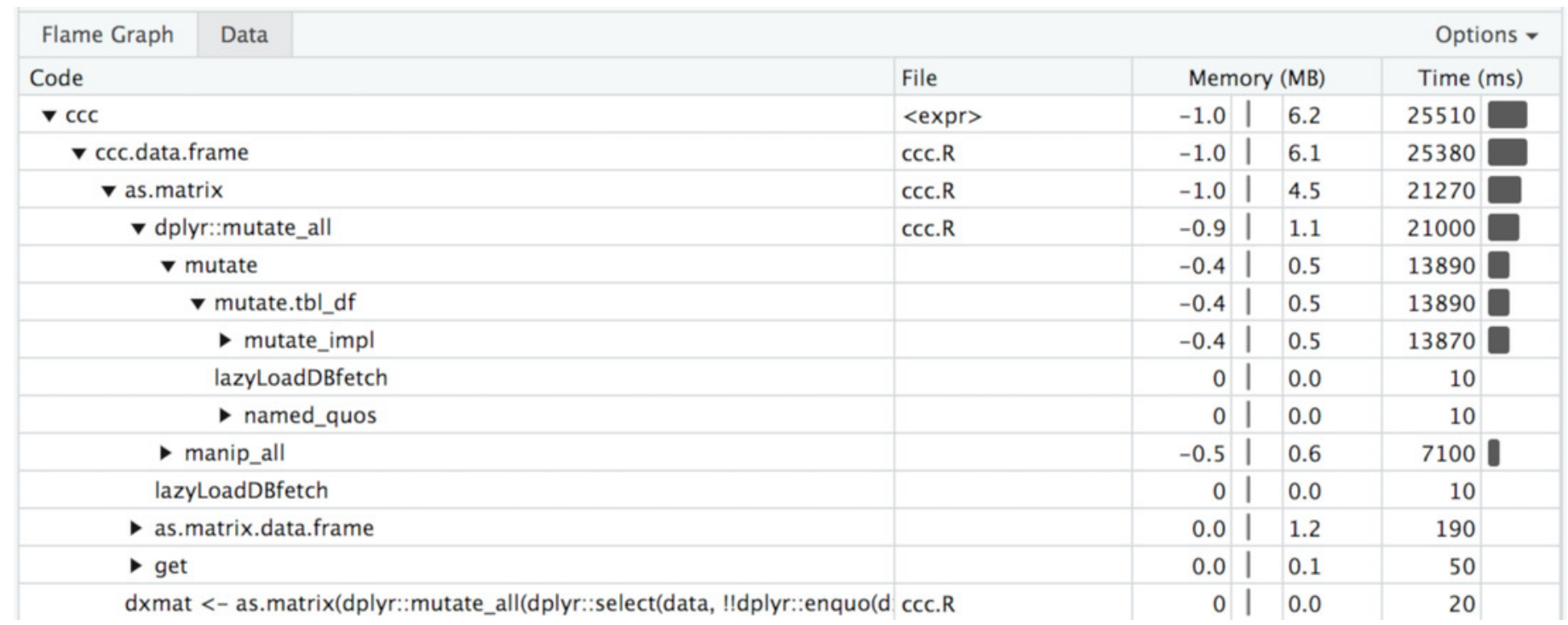




\section{Figure 7}

\section{Profvis Flame Graph .Call()}

Visual depiction of memory allocation/deallocation, execution time, and call stack; note the limitations in detail at the .Call() function where custom compiled code is called.

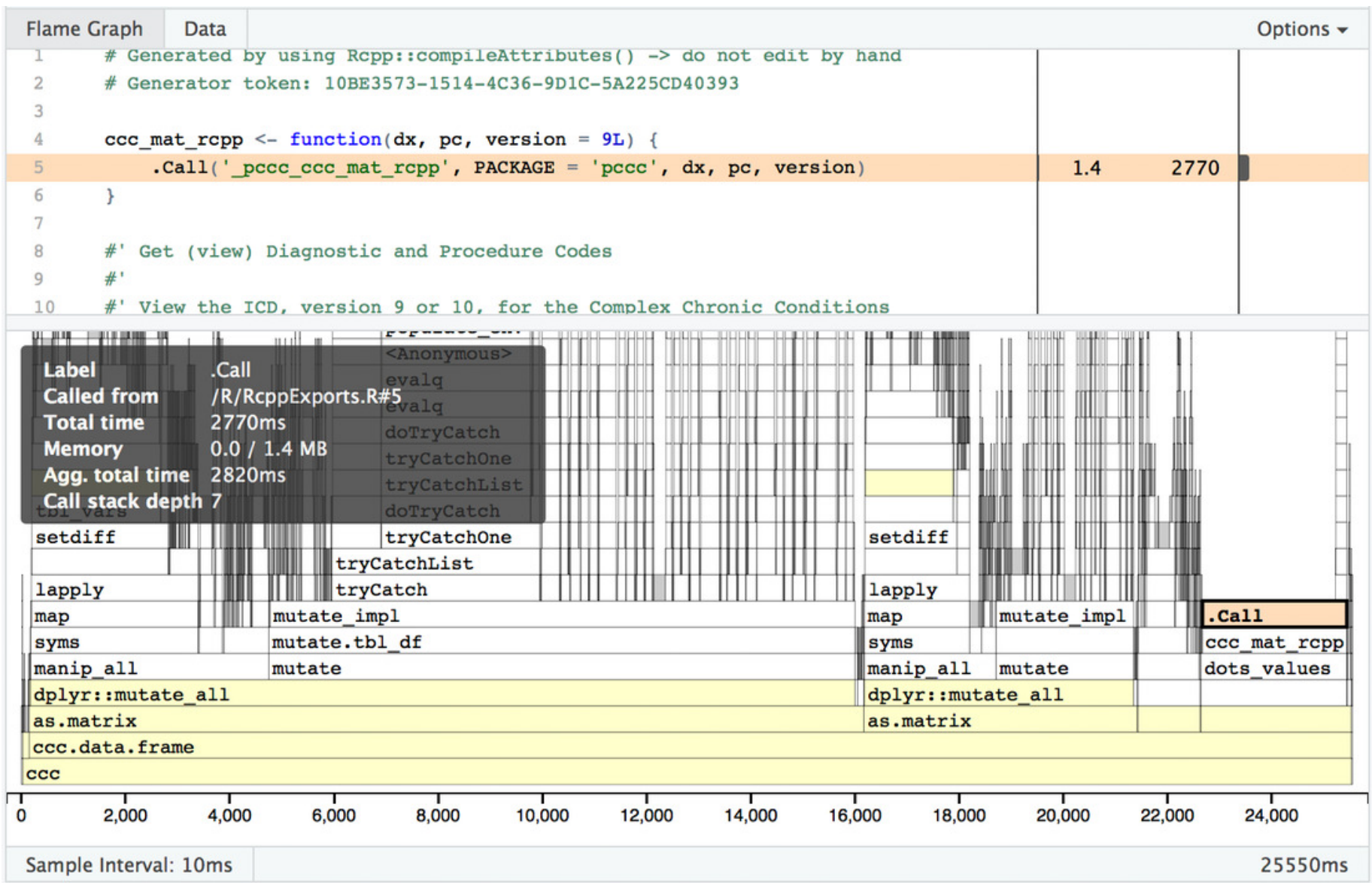

